\title{
Table of Contents / Table des matières
}

Editorial: Documents and Tools

RUTH BRADLEY-ST-CYR

From Threshold, to Living Room, to Kitchen:

On the Architectonics of Books

PATRICIA DEMERS

Canadian Comic Books at Library and Archives Canada

Revisiting the Library of Edward Gibbon

RICHARD VIRR

An Index of Nelson Ball's Little Magazines:

Volume 63 (1963-1967), Weed (1966-1967), and

Hyphid (1968)

CAMERON ANSTEE

Table of Contents: Testimony to the Royal Commission

on Book Publishing

RUDH BRADLEY-ST-CYR

The Writers' Union Meets the Royal Commission ARCHIVES OF ONTARIO, ROYAL COMMISSION FONDS

Books in Review / Comptes rendus

KRISTINE SMITKA and/et STÉPHANIE BERNIER

Micheline Cambron, Myriam Côté et Alex Gagnon, dir., Les journaux québécois d'une guerre à l'autre: Deux états de la vie culturelle québécoise au $X X^{e}$ siècle (Dominique Marquis)

Richard Dufour, Bibliothèque de l'Université Laval: 150 ans d'histoire, I852-20I7 (Marcel Lajeunesse)

Elyse Graham, The Republic of Games: Textual Cultures Between Old Books and New Media (Jon Saklofske) 
Marie Philip, Books that Matter (Peter Midgley)

188

Richard Landon, A Long Way from the Armstrong Beer Parlour: A Life in Rare Books: Essays by Richard Landon 192 (Bruce Whiteman)

Laura K. Davis and Linda M. Morra, eds., Margaret Laurence \& Jack McClelland, Letters (Janet B. Friskney)

Lise Jaillant, Cheap Modernism: Expanding Markets, Publishers' Series and the Avant-Garde

(David Buchanan)

Dean Irvine, Vanessa Lent, and Bart Vartour, eds., Making Canada New: Editing, Modernism, and New Media (Anna Sajecki)

François Séguin, D'obscurantisme et de lumières:

la bibliothèque publique au Québec des origines au $21^{e}$ siècle;

Claude Corbo (dir.), avec la collaboration de Sophie Montreuil et d'Isabelle Crevier, Bibliothèques québécoises remarquables 204 (Peter F. McNally, trans. by Marie-Claude Rochon)

François Séguin, D’obscurantisme et de lumières:

la bibliothèque publique au Québec des origines au $21^{e}$ siècle;

Claude Corbo (dir.), avec la collaboration de Sophie Montreuil et d'Isabelle Crevier, Bibliothèques québécoises remarquables 208 (Peter F. McNally)

Tony Tremblay, ed., New Brunswick at the Crossroads:

Literary Ferment and Social Change in the East

(Rachel Bryant)

David McKnight, Experiment: Printing the Canadian

Imagination (Gregory Betts)

Marie-Claire Boscq, Imprimeurs et libraires parisiens sous surveillance (I8I4-I848) (Anthony Glinoer)

Ad Stijnman and Elizabeth Savage, eds., Printing Colour I400-I700: History, Techniques, Functions and Receptions 218 (Ruth-Ellen St. Onge) 
Bart Layton (writer-director), American Animals (Linda Quirk)

Means and Purposes: A Suggestion to Our Members and Friends

'Qui veut la fin veut les moyens'

Information for Authors

Information à l'intention de nos collaborateurs

Call for Contributions: LGBTQ+ Print in Canada:

Overviews and Perspectives

Appel à contributions: L'imprimé LGBTQ+ au Canada et ailleurs : bilans et perspectives

Call for Reminiscences: Memorial section for William F.E. Morley, Francess Halpenny, and Greta Golick

Appel à contributions: Section commémorative en l'honneur de William F.E. Morley, Francess Halpenny et Greta Golick 